\title{
Nonshaved Ear Surgery: Effect of Hair on Surgical Site Infection of the Middle Ear/Mastoid Surgery and Patients' Preference for the Hair Removal
}

\author{
Dong-Hee Lee, Soonil Yoo, Eunhye Shin, and Yesun Cho \\ Department of Otolaryngology-Head and Neck Surgery, College of Medicine, The Catholic University of Korea, Seoul, Korea
}

\author{
Received February 23, 2018 \\ Revised March 21, 2018 \\ Accepted March 29, 2018
}

\begin{abstract}
Background and Objectives: This study aimed 1) to compare the rates of surgical site infection (SSI) between two groups with and without preoperative hair shaving, 2) to compare the bacterial colonization just before the skin incision between them, and 3) to evaluate people's preference for the hair shaving. Subjects and Methods: The retrospective study enrolled cases in which middle ear and mastoid surgery was performed with as well as without hair removal. Main measurement outcomes were the SSI rate within 3 months following the surgery, bacterial culture results obtained from the incision area just before the skin incision, and questionnaire to evaluate the preference for hair shaving from patients with chronic suppurative otitis media but without experience with the ear surgery. Results: This study did not show any difference in the rates of SSI and bacterial colonization between two groups with and without preoperative hair shaving. Most patients without experience with the ear surgery chose the nonshaved ear surgery, even though the questionnaire presented a comment as follow; "Your hair will always grow back as the growth speed of about $1.25 \mathrm{~cm}$ per month." Conclusions: There is no evidence showing that preoperative shaving of the surgical site is helpful for the SSI than no hair removal. Nonshaved middle ear and mastoid surgery via postauricular approach appears to be preferable. Contrary to doctors' popular belief, the hair shaving can cause psychological discomfort, especially for women. Now is the time to keep the balance between the professional's perspective and the patients' preferences.

J Audiol Otol 2018;22(3):160-166
\end{abstract}

KEY WORDS: Otologic surgical procedures · Hair removal · Preoperative care · Surgical wound infection.

\section{Introduction}

There has been several reports indicating that preoperative hair shaving before the operation increases the risk of surgical site infection (SSI) significantly than no hair removal due to the razor-induced microtrauma and breakdown of the skin surface barrier [1-4]. SSI after the middle ear and mastoid surgery contributes substantially to surgical morbidity and mortality. Postoperative wound infection can increase the length of hospital stay and lower the successful uptake of a graft. The prevention of SSI is the first and key step in its treatment

This is an Open Access article distributed under the terms of the Creative Commons Attribution Non-Commercial License (http://creativecommons.org/licenses/by-nc/4.0/) which permits unrestricted non-commercial use, distribution, and reproduction in any medium, provided the original work is properly cited.
[4]. This includes meticulous operative technique, timely and appropriate prophylactic antimicrobials, and preventive actions against bacterial contamination of the operative field.

Among three common skin incisions (transcanal, endaural, and postauricular) for the otological procedures including tympanomastoid surgeries, post- or retroaurcular incision has been most commonly used because it provides the best exposure and allows harvest a fascia in the same surgical field. The psychological distress of hair shaving may be quite significant for most patients, especially for women. Nevertheless, preoperative hair shaving has been a well-established practice among many otologic surgeons based on the belief that the hair shaving can prevent postoperative infections and facilitate good viewing of the operative field, secure attachment and/or easy removal of the drapes, concealing of annoying hairs, and clo- 
sure of the wound $[5,6]$. In addition, many surgeons think that the hair will always grow back and that patients should endure somewhat cosmetic inconvenience because it may be no doubt is a minor issue compared with the risk of postoperative wound infection.

The purposes of this study are: 1) to compare the rates of SSI between two groups with and without preoperative hair shaving and 2) to compare the bacterial colonization just before the skin incision between them. It also aimed 3) to evaluate patients' preference on hair shaving before the middle ear and mastoid surgery.

\section{Subjects and Methods}

\section{Study design}

The retrospective study enrolled all cases in which middle ear and mastoid surgery was performed without hair removal to cure chronic perforation of tympanic membrane as well as chronic suppurative otitis media with or without mastoiditis between March 2016 and August 2016. As control group, all cases in which middle ear and mastoid surgery was performed after preoperative hair removal by the same surgeon during the same period last year. All cases in both groups got the middle ear and mastoid surgery by one otologic surgeon, who got more than 10 years of experience performing otologic surgeries.

Exclusion criteria were: 1) patients of American Society of Anesthesiologists (ASA) Physical Status Classification of $>3$; 2) immunocompromised patients; 3) contaminated or dirty-infected ear at the time of the surgery; 4) patients with a history of radiation to head and neck region; and 5) patients who were followed postoperatively for less than 3 months.

\section{Classification of surgical site infection for the middle ear and mastoid surgery}

In defining the SSI, the infection of postauricular incision for the middle ear and mastoid surgery is classified as a deep incisional SSI, based on Centers for Disease Control (CDC) SSI classification system. This system classifies the infection of the ear and mastoid as a specific organ/space SSI [1]. Based on above definition, this study classified SSI for the middle ear and mastoid surgery into two. One is an infection that involves only skin or subcutaneous tissue of the incision (incisional SSI). The other is an infection of the graft or middle ear/mastoid (organ/space SSI). The criteria of the former SSI were: 1) infection occurring within 30 days after the operation if no implant is left in place, and the infection appears to be related to the operation; 2) infection involving deep soft tissues (e.g., fascial and muscle layers) of the incision; and 3) at least one of the following: a) purulent drainage from the deep incision but not from the organ/space component of the surgical site; b) a deep incision spontaneously dehisces or is deliberately opened by a surgeon when the patient has at least one of the following signs or symptoms: fever $\left(>38^{\circ} \mathrm{C}\right)$, localized pain, or tenderness, unless site is culture-negative; c) an abscess or other evidence of infection involving the deep incision is found on direct examination, during reoperation, or by histopathologic or radiologic examination; and d) diagnosis of a deep incisional SSI by a surgeon or attending physician. The latter SSI was defined as: 1) organisms isolated from an aseptically obtained culture of purulent drainage from the ear canal, even though clean skin or subcutaneous tissue of the incision; and 2) pulsating, edematous graft found on direct examination.

\section{Skin preparation and hair shaving}

Preoperative patient care and prophylactic antimicrobial agent through the intravenous route were performed in the same fashion in both groups. In the control group with hair shaving, each patient shampooed his or her hair with commercial shampoo the night before the operation. The postauricular area was shaved with a dry razor. In the study group without hair shaving, each patient shampooed the hair with commercial shampoo the night before the operation and wore a plastic cap to fully cover hair during the sleep. He or she shampooed the hair with commercial shampoo 1-2 hours before moving to the operating room.

Preoperative painting and draping were performed in the same fashion in both groups (Fig. 1). Before the operation started, the entire surgical field was painted with $10 \%$ povidone-iodine solution and then $70 \%$ isopropyl alcohol. Excess solution was cleaned with sterile gauze, and the painted field was then allowed to dry. The surgical field was isolated with sterile drapes. A transparent adhesive drape was placed over the skin to be incised (Fig. 2).

All surgical wounds were closed in the same manner; simple interrupted vicryl periosteal closure, simple interrupted vicryl fascial closure and simple interrupted vicryl subcutaneous closure to approximate the skin edges. Skin incision was closed using Histoacryl ${ }^{\circledR}$ topical skin adhesive (B. Braun Surgical SA, Rubi, Spain). Drain was not used in all cases. Mastoid packing was applied using gelatin sponge (Spon$\operatorname{gostan}^{\mathrm{TM}}$; Ethicon, Somerville, NJ, USA).

\section{Data collection and analysis}

The medical records included demographic data, SSI within 3 months following operation, and any associated factors with the SSI. All culture specimens were obtained by 

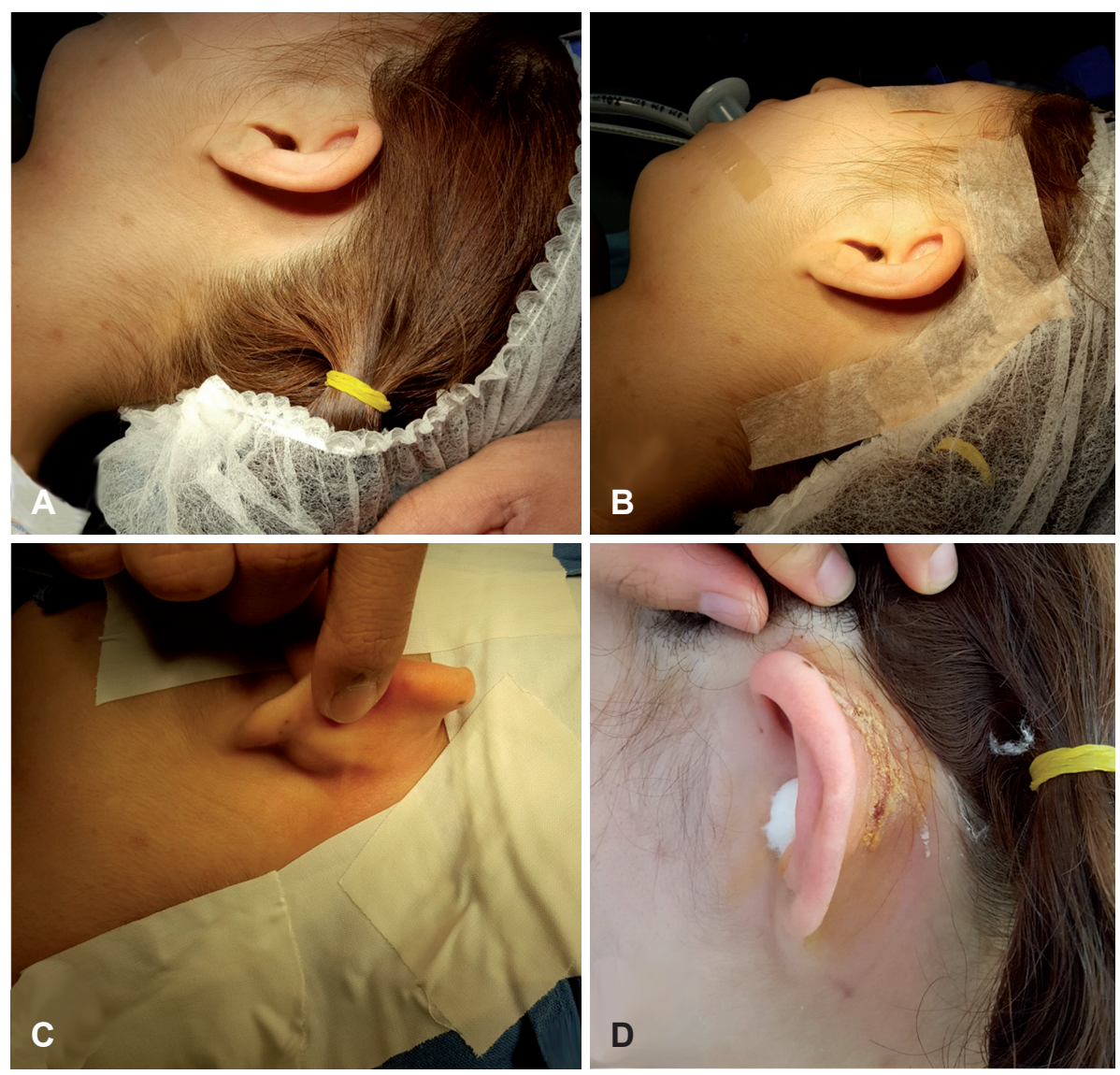

Fig. 1. 17 year-old female. A-C: Preparation of nonshaved ear for postauricular tympanoplasty. D: 1st postoperative day.

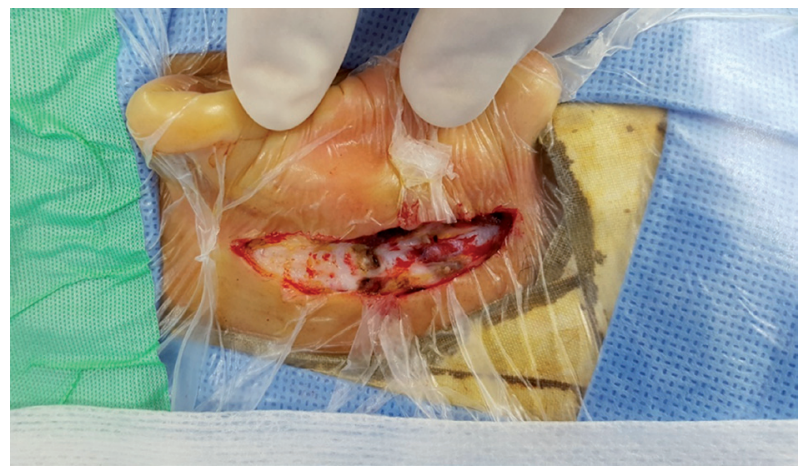

Fig. 2. Draping and skin incision for nonshaved ear surgery. Skin incision was done through a transparent adhesive drape, which was placed over the skin to be incised.

standardized technique. Cultures were obtained with a standard cotton-tipped culture swab. In both groups, each culture swab was swabbed over expected skin incision area for 1 to 2 seconds after the painting and draping and transported in an aerobic or anaerobic transport media tube.

To find out patient's preference for the hair shaving, the questionnaire was surveyed to 60 patients (30 men and 30 women; 5 participants per each decade from 20 s to 70 s) who visited our outpatient clinic for chronic suppurative otitis media. Two photos of side view (with and without hair shav- ing) were taken from real patients; one photo was taken while his or her hairs were left naturally and the other was taken while his or her hairs were put aside to show postauricular area maximally. These 2 photos were taken from 3 men and 3 women of various ages in each group. On a questionnaire, 12 photos of patients with hair shaving and 12 photos without hair shaving were presented together with a comment as follow; "Your hair will always grow back as the growth speed of about $1.25 \mathrm{~cm}$ per month."

Data were described using the mean \pm standard deviation. Statistical analysis was performed by using IBM-SPSS statistics software (version 22, IBM Corp., Armonk, NY, USA). $p$-values less than 0.05 were considered to be significant.

\section{Ethics statement}

The study was approved by the Institutional Review Board (IRB \#UC16RISI0142). The informed consent for the clinical study was waived because of retrospective chart review.

\section{Results}

Epidemiologic data and results of main outcome parameters (pre-incisional bacterial colonization \& SSI) are summa- 
rized in Table 1. There was no significant difference in gender, age, pre-incisional bacterial colonization and SSI rate between nonshaved and shaved groups.

Although pre-incisional bacterial colonization was found in 2 cases of nonshaved group, there was no SSI of skin incision or middle ear/mastoid during 3-month F/U in both groups. One case was a 52-year old woman who got canal wall-up tympanomastoidectomy for adhesive otitis media, in which Staphylococcus hominis was identified. The other was a 69-

Table 1. Summary of descriptive statistics

\begin{tabular}{|c|c|c|}
\hline & $\begin{array}{c}\text { Study } \\
\text { group } \\
\text { (unshaved) } \\
n=50\end{array}$ & $\begin{array}{c}\text { Control } \\
\text { group } \\
\text { (shaved) } \\
n=66\end{array}$ \\
\hline Gender (male:female) & $20: 30$ & $24: 42$ \\
\hline Age (mean $\pm S D$, years-old $)$ & $49.5 \pm 19.4$ & $46.3 \pm 19.4$ \\
\hline Pre-incision bacterial colonization & 2 cases & 0 case \\
\hline \multicolumn{3}{|l|}{ Surgical site ilnfection } \\
\hline $\begin{array}{l}\text { 1) Skin or subcutaneous tissue } \\
\text { of the incision }\end{array}$ & 0 case & 1 case \\
\hline 2) Graft or middle ear/mastoid & 0 case & 0 case \\
\hline
\end{tabular}

year old man who got canal wall-up tympanomastoidectomy for chronic suppurative otitis media, in which Staphylococcus epidermidis was identified.

Fig. 3 showed two examples of draped operative field and 1 st postoperative day in nonshaved group. If a hair line was close to postauricular sulcus, it was somewhat difficult to conceal the hair and expose the operative filed properly but the exposure was enough to do tympanoplasty or tympanomastoidectomy.

Questionnaire survey results showed the patient's preference for the hair shaving prior to the surgery. Most of questioned persons ( 25 of 30 men \& 29 of 30 women) chose the nonshaved ear surgery, if they will get the middle ear and mastoid surgery. Ninety percent of total participations in the questionnaire survey showed the preference for the nonshaved ear surgery.

\section{Discussion}

This study demonstrated that there was no difference in the risk of SSI rates between two groups with and without hair shaving. For a long time, hair to accommodate the antic-

Fig. 3. Photos of nonshaved ear. Images of operative field (A) and 1st postoperative day (B) in a case of 18 year-old female having canal walldown tympanomastoidectomy. Images of operative field (C) and 1st postoperative day (D) in a case of 19 yearold male having tympanoplasty.
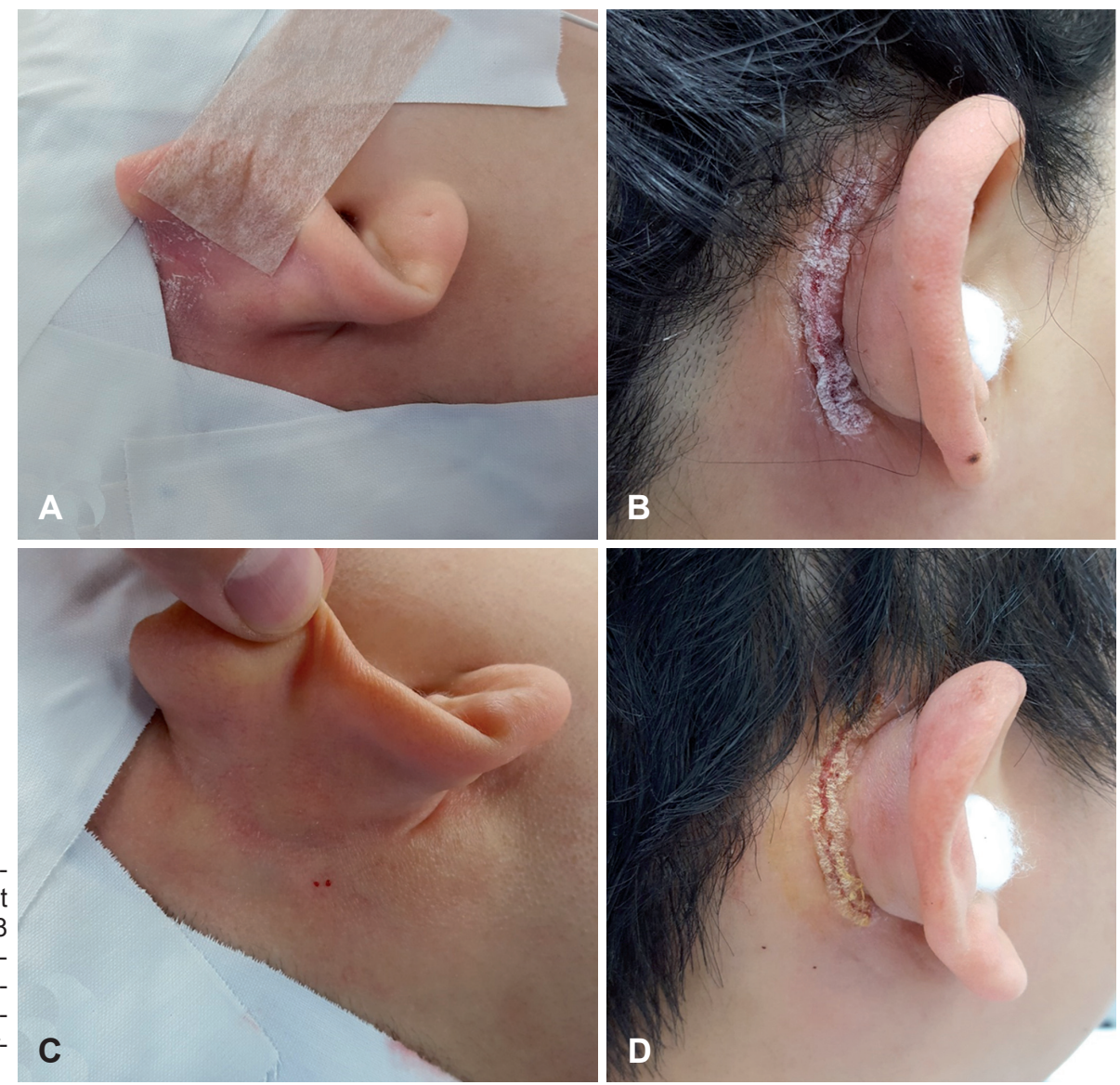
ipated incision and extent of the surgery has been ill-conceived to be associated with poor cleanliness and higher risk of SSIs. For clean or clean-contaminated wounds, preoperative preparation of the surgical site aims to reduce the colonization of the surgical wound by normal skin flora. To do this, many surgical textbooks describe that adequate clipping of hair at the area of the anticipated incision(s) should be done $[5,6]$. They do not recommend preoperative hair shaving because shaving with a razor destructs the skin barrier and increases the risk of SSI. They also emphasize that to cleanse the skin with isopropyl alcohol after painting with povidone iodine is very important because it helps to reduce the bacterial count and remove skin oil. The "Guideline for Prevention of Surgical Site Infection, 1999" from CDC stated that hair shaving or clipping immediately before the operation reduced the risk of SSI [1]. World Health Organization (WHO) reported recently the "Global Guidelines for the Prevention of Surgical Site Infection," which recommends that hair shaving is strongly discouraged at all times for patients undergoing any surgical procedure, whether preoperatively or in the operating room [2,3]. New WHO recommendations state that for patients undergoing any surgical procedure, hair should not be shaved whether preoperatively or in the operating room but, if absolutely necessary, can be removed only with a clipper $[2,3]$. They recommend bathing or taking a shower using either plain soap or an antimicrobial soap prior to surgery for patients.

For the hair removal using either shaving or clipping might be necessary to facilitate adequate exposure and preoperative planning of the skin incision, many surgeons prefer preoperative hair removal. However, in cases of usual middle ear and mastoid surgery including tympanomastoidectomy, where postauricular incision is usually placed about $1 \mathrm{~cm}$ behind the postauricular crease, anticipated incised and operated area can be fully exposed by meticulous and nice attachment of adhesive plaster (Fig. 1). Even if the skin incision is placed more posteriorly in cases of canal wall-down tympanomastoidectomy, nonshaved surgical field is not a problem to surgeons.

The guidelines of association of surgical technologists in USA state that based on the surgeon's orders, the patient takes a shower with an antiseptic agent the night before surgery and/or the morning of surgery. After shower with plain soap and shampoo, the patient must make sure that they are thoroughly rinsed off and then clean the skin of the surgical area with chlorhexidine gluconate. The patient should be instructed not to use a body lotion after showering with chlorhexidine gluconate. If the hair removal is needed, the skin and hair should be wetted in order to perform a wet shave preparation and the hair removal should be performed using a clipper as close to the time of surgery as possible. If hair removal is not performed, these guidelines suggest that it is an alternative method to apply a non-flammable gel to the hair but we use the adhesive plaster for keeping the hair out of the surgical wound [7]. Above guidelines on preoperative shower with soap as well as preparation with chlorhexidine gluconate are in accordance with those of new WHO recommendations [2,3].

From our experiences, surgical site skin preparation with alcohol is very important. New WHO recommendations told that alcohol-based antiseptic solutions for surgical site skin preparation are more effective than aqueous solutions in reducing the risk of SSI $[2,3]$. Ten percentage povidone-iodine solution used for surgical site skin preparation in our hospital is made in an aqueous solution. Therefore, second skin preparation with $70 \%$ isopropyl alcohol wash out the hair strands to accommodate the surgical site, which are stuck together due to sticky povidone-iodine aqueous solution.

One of the interesting results in this study was that most people wanted the nonshaved ear surgery even though they knew that the hair always grows back within 1-2 months. From our experiences, most patients who have the surgery ahead asked the necessity of hair shaving to doctors, which meant that the hair shaving was a cause of concern for them, especially women. This result is in accordance with others' findings [8-10]. From our view, the hair-removed area behind the auricle can be partially covered by surrounding hairs even in cases with hair shaving (Fig. 4) but the view of concerned people was not different from ours. Contrary to patients' opinion, many surgeons have put more weight on oldfashioned belief that the hair shaving prevents postoperative SSI and presents better operative filed than on patients' psychological distress or cosmetic inconvenience.

This study aimed to find out patients' preferences or values for surgeons to make a policy on how the skin is prepared for the middle ear/mastoid surgery through postauricular skin incision, which was the main strength of our study. Sometimes, medical paternalism really serves the patient best because it is generally true that patients lacking professional information may not make a sound medical decision. For much of traditional medical practice has been based on unequal power relation between patient and doctor, it has been felt that unmediated paternalism can no longer serve nowadays and patients' involvement has grown in the development of clinical practice guidelines. This concept has been reflected in making clinical practice guidelines, which are actually changing to adapt both the professional's perspective and the patients' preferences equally [11,12].

This study defined two types of SSI; one was incisional 

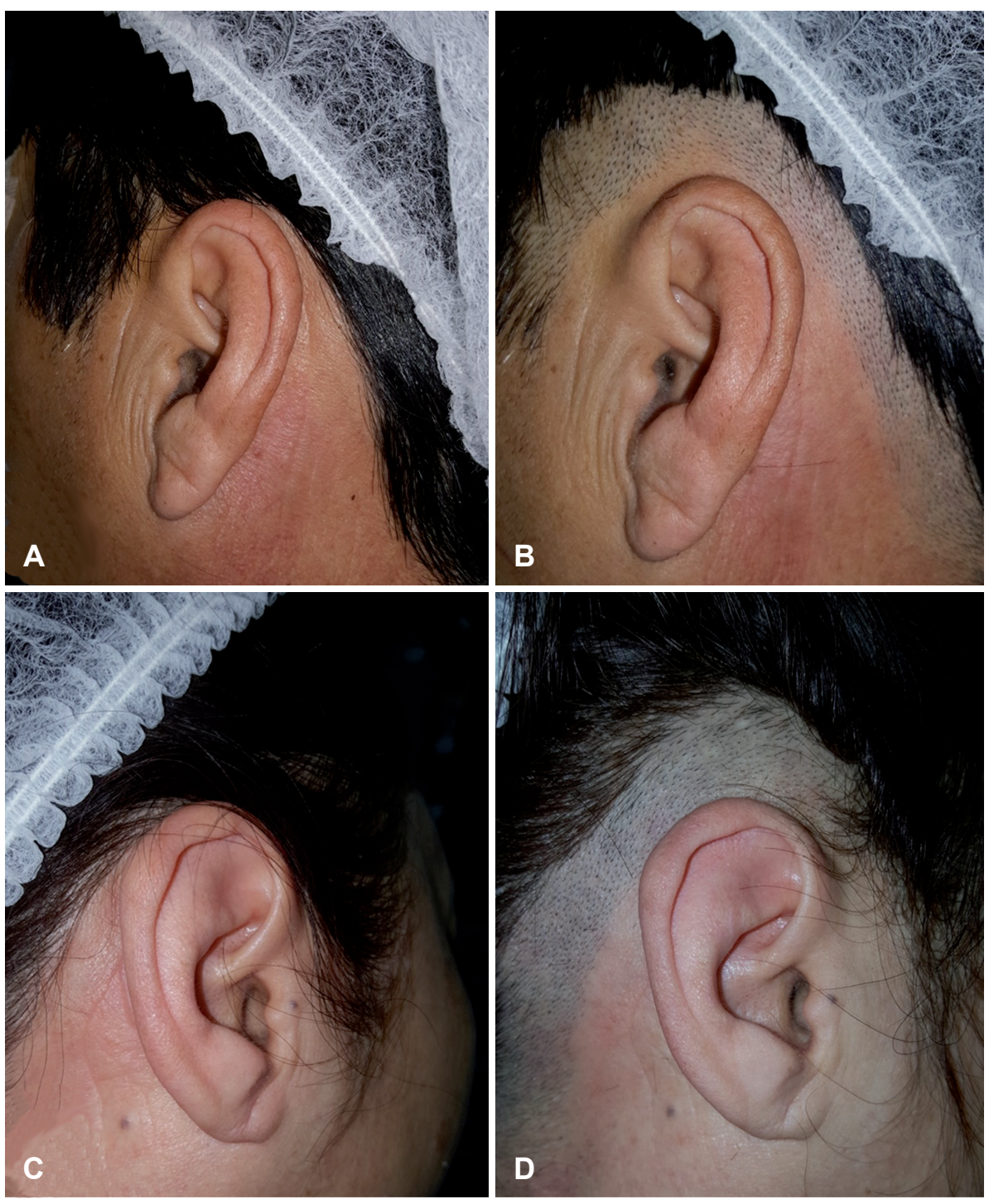

Fig. 4. Two sample photos of shaved ear for the middle ear and mastoid surgery. Images of before (A) and after (B) hair shaving in a man. Images of before (C) and after (D) hair shaving in a woman.

SSI and the other organ/space SSI. The limitation of this study was vague clinical definition of the latter organ/space SSI. Bastier, et al. [13] pointed out the same problems as we did. If the infected discharge from deep portion underneath the postauricular incision, deep incisional and organ/space SSI may be not easily differentiated because of possible mastoid-subcutaneous fistula, especially in the tympanomastoidectomy cases. It is also difficult to specify the origin of purulent postoperative otorrhea because it may be due to infection either of the ear canal incision (incisional SSI) or of the graft or middle ear (organ/space SSI).

In conclusion, the risk of SSI rates was not different between two groups with and without hair shaving in this study. Avoiding hair shaving in the middle ear and mastoid surgery via postauricular approach would appear to be preferable. Contrary to doctors' popular belief, hair shaving can cause psychological discomfort, especially for women. This means that it is necessary for doctors to read patients' mind and need. More clinical research will be needed to build evidences to make clinical practice guidelines in such a way as to balance the doctors' perspective and the patients' preferences equally.

\section{Conflicts of interest}

The authors have no financial conflicts of interest.

\section{REFERENCES}

1) Mangram AJ, Horan TC, Pearson ML, Silver LC, Jarvis WR.; Centers for Disease Control and Prevention (CDC) Hospital Infection Control Practices Advisory Committee. Guideline for prevention of surgical site infection, 1999. Am J Infect Control 1999;27:97-132; quiz 133-4; discussion 96.

2) Allegranzi B, Bischoff P, de Jonge S, Kubilay NZ, Zayed B, Gomes $\mathrm{SM}$, et al. New WHO recommendations on preoperative measures 
for surgical site infection prevention: an evidence-based global perspective. Lancet Infect Dis 2016;16:e276-87.

3) World Health Organization. Global guidelines for the prevention of surgical site infection. Geneva: World Health Organization Press; 2016.

4) Reichman DE, Greenberg JA. Reducing surgical site infections: a review. Rev Obstet Gynecol 2009;2:212-21.

5) Jackson CG. Principles of temporal bone and skull base surgery. In: Glasscock ME, Gulya AJ, editors. Glasscock-Shambaugh surgery of the ear. 5th ed. Hamilton (OT): BC Decker Inc;2003. p.263-88.

6) Fayad JN, Hoskins BS, Benecke EJ Jr. Otologic instrumentation. In: Brackmann DE, Shelton C, Arriaga MA, editors. Otologic surgery. 3rd ed. Philadelphia (PA): Saunders;2010. p.1-20.

7) Association of Surgical Technologists. Surgical technology for the surgical technologist: a positive care approach. 4th ed. Clifton Park (NY): Delmar;2012.

8) Kretschmer T, Braun V, Richter HP. Neurosurgery without shaving: indications and results. Br J Neurosurg 2000;14:341-4.

9) Gil Z, Cohen JT, Spektor S, Fliss DM. The role of hair shaving in skull base surgery. Otolaryngol Head Neck Surg 2003;128:43-7.

10) Kose G, Tastan S, Kutlay M, Bedir O. The effects of different types of hair shaving on the body image and surgical site infection in elective cranial surgery. J Clin Nurs 2016;25:1876-85.

11) van der Weijden T, Légaré F, Boivin A, Burgers JS, van Veenendaal $\mathrm{H}$, Stiggelbout AM, et al. How to integrate individual patient values and preferences in clinical practice guidelines? A research protocol. Implement Sci 2010;5:10.

12) Rashid A, Thomas V, Shaw T, Leng G. Patient and public involvement in the development of healthcare guidance: an overview of current methods and future challenges. Patient 2017;10:277-82.

13) Bastier PL, Leroyer C, Lashéras A, Rogues AM, Darrouzet V, Franco-Vidal V. Early and late surgical site infections in ear surgery. Acta Otorhinolaryngol Ital 2016;36:127-34. 